ఠ

\title{
Indirubin inhibits cell proliferation, migration, invasion and angiogenesis in tumor-derived endothelial cells
}

This article was published in the following Dove Press journal:

OncoTargets and Therapy

\section{Zhuohong Li \\ Chaofu Zhu \\ Baiping An \\ Yu Chen \\ Xiuyun $\mathrm{He}$ \\ Lin Qian \\ Lan Lan \\ Shijie Li}

Department of Oncology, The Affiliated Hospital of Chengdu University of Traditional Chinese Medicine, Chengdu, Sichuan, China
Correspondence: Shijie Li Department of Oncology, The Affiliated Hospital of Chengdu University of Traditional Chinese Medicine, 39\# I2 Bridge Road, Jinniu District, Chengdu, Sichuan Province 610072, China

$\mathrm{Tel} / \mathrm{fax}+862887765649$

Email chinajef@I26.com
Purpose: Hepatocellular carcinoma is one of the most predominant malignancies with high fatality rate and its incidence is rising at an alarming rate because of its resistance to radio- and chemotherapy. Indirubin is the major active anti-tumor ingredient of a traditional Chinese herbal medicine. The present study aimed to analyze the effects of indirubin on cell proliferation, migration, invasion, and angiogenesis of tumor-derived endothelial cells (Td-EC).

Methods: Td-EC were derived from human umbilical vein endothelial cells (HUVEC) by treating HUVEC with the conditioned medium of human liver cancer cell line HepG2. Cell proliferation, migration, invasion, and angiogenesis were assessed by MTT, wound healing, in vitro cell invasion, and in vitro tube formation assay.

Results: Td-EC were successfully obtained from HUVEC cultured with 50\% culture supernatant from serum-starved HepG2 cells. Indirubin significantly inhibited Td-EC proliferation in a dose- and time-dependent manner. Indirubin also inhibited Td-EC migration, invasion, and angiogenesis. However, indirubin's effects were weaker on HUVEC than Td-EC.

Conclusion: Indirubin significantly inhibited Td-EC proliferation, migration, invasion, and angiogenesis.

Keywords: indirubin, Td-EC, proliferation, migration, invasion, angiogenesis

\section{Introduction}

Hepatocellular carcinoma (HCC) is the most common primary liver cancer and the second most frequent cause of cancer-related deaths worldwide. ${ }^{1}$ Tumor occurrence and development are very complicated processes. Cancer cells stimulate angiogenesis in an effort to maintain and allow tumor progression. ${ }^{2}$ Proliferation, migration, and activation of endothelial cells are involved in tumor angiogenesis. ${ }^{3}$ Therefore, tumor-derived endothelial cells (Td-EC) are considered to be ideal therapeutic targets in antiangiogenic therapies. Tumor endothelial markers (TEMs) showed elevated expression during tumor angiogenesis than in normal angiogenesis. TEM1 shows overexpression in tumor tissues and is engaged in tumor invasion, growth, angiogenesis, and microvasculature maturation. ${ }^{4-6}$ TEM8 shows a potential role in cell adhesion, migration, and tubule formation. ${ }^{7,8}$

Current therapeutic modalities for HCC include curative options such as surgical resection, liver transplantation, and local ablation or palliative procedures such as catheter-directed therapies and systemic therapy. ${ }^{9}$ However, HCC mortality rate is very high because it is quite resistant to radio- and chemotherapies. ${ }^{10}$ Thus, new treatment choices are critically required. 
Traditional Chinese Medicine (TCM) is a valuable resource that includes many biologically active components that are both safe and effective. Indirubin, extracted from indigo and an active ingredient of Danggui Longhui Wan formula, is the first TCM extract shown to be effective in the treatment of chronic myelogenous leukemia. ${ }^{11}$ However, the effects of indirubin on Td-EC have not been addressed.

In the present study, we investigated the effects of indirubin on cell proliferation, migration, invasion, and angiogenesis of Td-EC to explore new approaches in cancer therapy.

\section{Materials and methods}

\section{Reagents}

Indirubin (purity $\geq 98 \%$ ) was purchased from Shanghai Yuan Ye Biological Technology Co., Ltd. (Shanghai, China) and dissolved in dimethylsulfoxide (DMSO, Kelong Co, Chengdu, China). Roswell Park Memorial Institute (RPMI) 1640 Medium (PRMI1640, Thermo Fisher Scientific, Waltham, MA, USA) and fetal bovine serum (FBS) were purchased from Thermo Fisher Scientific. The $0.25 \%$ trypsin was purchased from HyClone (Shanghai, China). Matrigel was purchased from Becton Drive (BD, Franklin Lakes, NJ, USA).

\section{Cell culture}

Human umbilical vein endothelial cells (HUVEC) line was obtained from the Type Culture Collection of the Chinese Academy of Sciences (Shanghai, China). Cells were grown in Dulbecco's Modified Eagle's Medium (DMEM, Thermo Fisher Scientific) containing 20\% FBS, $10 \mathrm{ng} / \mathrm{L}$ vascular endothelial growth factor (VEGF) (Thermo Fisher Scientific), and $1 \%$ antibiotics (100 units $/ \mathrm{mL}$ penicillin, $100 \mathrm{mg} / \mathrm{mL}$ streptomycin, Shanghai Canspec Scientific Instruments Co., Ltd., Shanghai, China) under standard conditions in a humidified incubator at $37^{\circ} \mathrm{C}$ with $5 \% \mathrm{CO}_{2}$.

Human HCC cell line HepG2 cells were obtained from the American Type Culture Collection (ATCC, Manassas, VA, USA) and maintained in RPMI 1640 medium with 10\% FBS and $1 \%$ antibiotics under standard conditions. HepG2 cells were cultured in serum-free RPMI 1640 for 48 hours when they reached $70 \%$ confluence. Then, the medium from the serumstarved HepG2 cells was collected, filtered by $0.22 \mu \mathrm{m}$ filter membrane, and stored at $-80^{\circ} \mathrm{C}$ as the conditioned medium.

HUVEC were cultured with the complete medium with $50 \%$ of the HepG2-conditioned medium for 48 hours to become Td-EC. The identity of Td-EC were validated by checking TEMs that are associated with tumor-specific angiogenesis and are potentially useful to distinguish between tumor and normal endothelium. ${ }^{12}$ In this study, TEM1 and TEM8 were chosen for Td-EC detection.

\section{RNA isolation and reverse transcription- polymerase chain reaction (RT-PCR)}

Total RNA was extracted from Td-EC and HUVEC using Trizol Reagent (Thermo Fisher Scientific), and cDNA was synthesized from total RNA using RevertAid First Strand cDNA Synthesis Kit (Thermo Fisher Scientific). The conditions for PCR were 30 cycles of: denaturation $\left(95^{\circ} \mathrm{C} / 30 \mathrm{~s}\right)$, annealing $\left(68^{\circ} \mathrm{C} / 30 \mathrm{~s}\right)$, extension $\left(72^{\circ} \mathrm{C} / 1 \mathrm{~min}\right)$, and final extension $\left(72^{\circ} \mathrm{C} / 10 \mathrm{~min}\right)$ using Deam Taq PCR Master Mix (2X) (Thermo Fisher Scientific). TEM1 and TEM8 gene expression was detected by PCR with normalization by $\beta$-actin level as an internal control. Each experiment was performed in triplicate. The sequences of the primers used in this study were: forward primer $5^{\prime}$-ctgatgggtgaggtctggtt-3' and reverse primer $5^{\prime}$-cattatcccaactgcccagc- $3^{\prime}$ for TEM1; forward primer $5^{\prime}$-gtctcctcetggcagaactt- $3^{\prime}$ and reverse primer $5^{\prime}$-gctgcaccactggaatgaaa-3' for TEM8; forward primer $5^{\prime}$-cctctatgccaacacagtgc- $3^{\prime}$ and reverse primer $5^{\prime}$-cctgcttgctgatccacatc- $3^{\prime}$ for $\beta$-actin.

\section{Cell proliferation assay}

To test the effect of indirubin on cell proliferation, a quantitative colorimetric assay with MTT (Thermo Fisher Scientific) was applied. Briefly, HUVEC and Td-EC were seeded onto 96-well plates at $5 \times 10^{3}$ cells in $100 \mu \mathrm{L}$ of medium per well. After incubation with $5,10 \mu \mathrm{M}$ of indirubin for 24,48 , 72 hours, the cells were incubated with $0.5 \mathrm{mg} / \mathrm{mL}$ of MTT for 4 hours at $37^{\circ} \mathrm{C}$ in darkness. After removing the culture medium, $100 \mu \mathrm{L}$ of DMSO was added to dissolve formazan crystals within the cells. The absorbance was then measured using Synergy ${ }^{\mathrm{TM}}$ Microplate Reader (BioTek Instruments, Winooski, VT, USA) at a wavelength of $490 \mathrm{~nm}$. The cell proliferation rate (\%) was calculated using the following formula: (absorbance of experimental well/absorbance of control) $\times 100 \%$. Three replicate wells were used for each analysis.

\section{Cell migration assay}

HUVEC and Td-EC $\left(4 \times 10^{5}\right.$ cells/well $)$ were plated onto 6-well plates to reach $90 \%$ confluence. A wound was created by manually scraping the cell monolayer with a p1000 pipette tip. Then, cells were treated with 0,5 , and $10 \mu \mathrm{mol} / \mathrm{L}$ of indirubin for 48 hours. The cells were washed once with $1 \mathrm{~mL}$ of DMEM, which was then replaced with $2 \mathrm{~mL}$ of DMEM. Dishes were incubated at $37^{\circ} \mathrm{C}$ in a humidified 
atmosphere containing $5 \% \mathrm{CO}_{2}$. The cells were observed 48 hours later with an inverted fluorescence microscope (Leica DMI4000B, Bensheim, Germany) equipped with an Olympus Qcolor 3 digital camera (the same microscope was used for taking all micrographs). Cell migration was evaluated by migration potential (\%), which was the distance the cells migrated from the original wound margin. All the images were processed with ImagePro Plus (Media Cybernetics, Rockville, MD, USA). The experiments were repeated three times.

\section{In vitro tube formation assay}

Serum-free culture medium and pre-cooled melted Matrigel $(10 \mathrm{mg} / \mathrm{mL}, \mathrm{BD})$ were mixed at a ratio of 2:1 (v/v). A 24-well plate was coated with the Matrigel mixture, which was then allowed to polymerize for 1 hour at $37^{\circ} \mathrm{C}$. HUVEC and Td-EC $\left(1.5 \times 10^{5}\right.$ cells/well $)$ were seeded on the surface of the Matrigel at $37^{\circ} \mathrm{C}$ for 12 hours and tube formation was observed every 4 hours with an inverted fluorescence microscope (Leica DMI4000B) equipped with an Olympus Qcolor 3 digital camera. Changes in tube formation were observed, after adding $5 \mu \mathrm{M}$ of indirubin for 48 hours, under a microscope, and photographed at $100 \times$ magnification. Quantification of the tube formation was evaluated by the number of tube formations. The experiments were repeated three times.

\section{In vitro cell invasion assay}

Invasion of endothelial cells was tested using the Matrigelcoated Transwell system ( $8 \mu \mathrm{m}$ pore size and $6.5 \mathrm{~mm}$ diameter) in 24-well plates (Corning Costar, Cambridge, MA, USA). The lower chamber was filled with $500 \mu \mathrm{L}$ of DMEM medium with 10\% FBS. HUVEC and Td-EC $\left(1 \times 10^{5}\right.$ cells/well) were seeded into the upper chamber in serum-free media overnight. The cells were then fixed with methanol and stained with H\&E (Sigma, Shanghai, China). The cells on the upper filter surface were removed, and cell invasion was determined using an inverted fluorescence microscope (Leica DMI4000B) equipped with an Olympus Qcolor 3 digital camera at $100 \times$ magnification, by counting the cells that had migrated to the lower filter side. Samples were assayed twice in triplicate.

\section{Statistical analysis}

All experiments were repeated at least three times and all numerical data are presented as mean \pm standard error the mean. To evaluate the significant differences between the two groups, the means were compared using Student's $t$-test. $P<0.05$ was considered as statistically significant.
These analyses were performed using SPSS 13.0 software (SPSS, Chicago, IL, USA).

\section{Results \\ Td-EC induction and validation}

To determine whether HUVEC were induced to become Td-EC by HepG2 conditioned medium, TEM1 and TEM 8 were detected by PCR. We found that TEM1 and TEM8 were expressed in Td-EC but not in HUVEC (Figure 1A). Cell morphology showed that Td-EC appeared with endothelial cell morphological features (Figure 1B-D).

\section{Effects of indirubin on cell proliferation of Td-EC}

We found that indirubin inhibited cell proliferation of HUVEC and Td-EC in time- and dose-dependent manners (Figure 2). Compared to untreated HUVEC and Td-EC, indirubin at doses of 5 and $10 \mu \mathrm{M}$ significantly inhibited Td-EC proliferation, whereas indirubin's effects were weaker on HUVEC than Td-EC (Figure 2).

\section{Effects of indirubin on Td-EC migration}

Compared to untreated HUVEC and Td-EC, cell migration of Td-EC treated with indirubin for 48 hours was suppressed in a dose-dependent manner (Figure $3 \mathrm{~A}-\mathrm{L}$ ). The migration ability of Td-EC was stronger than HUVEC. However, the inhibitory effects of indirubin were much stronger on Td-EC than HUVEC. Quantification of the wound-healing assay by migration potential (\%) is shown in Figure 3M.

\section{Effects of indirubin on Td-EC angiogenesis in vitro}

Vascular tube formation ability was markedly stronger in Td-EC than HUVEC (Figure 4A and C). The vascular tube formation ability in Td-EC was significantly inhibited by indirubin, but the inhibition was less obvious in HUVEC (Figure 4A-D). Quantification of tube formation number $(\%)$ is shown in Figure 4E.

\section{Effects of indirubin on Td-EC invasion in vitro}

Transwell assays showed that indirubin had no effects on HUVEC migration (Figure 5A, B and E), whereas the invasion cell number of Td-EC was decreased significantly by indirubin treatment (Figure 5C-E). The invasion cell number of Td-EC was much more than that of HUVEC in the absence of indirubin treatment (Figure 5E). 

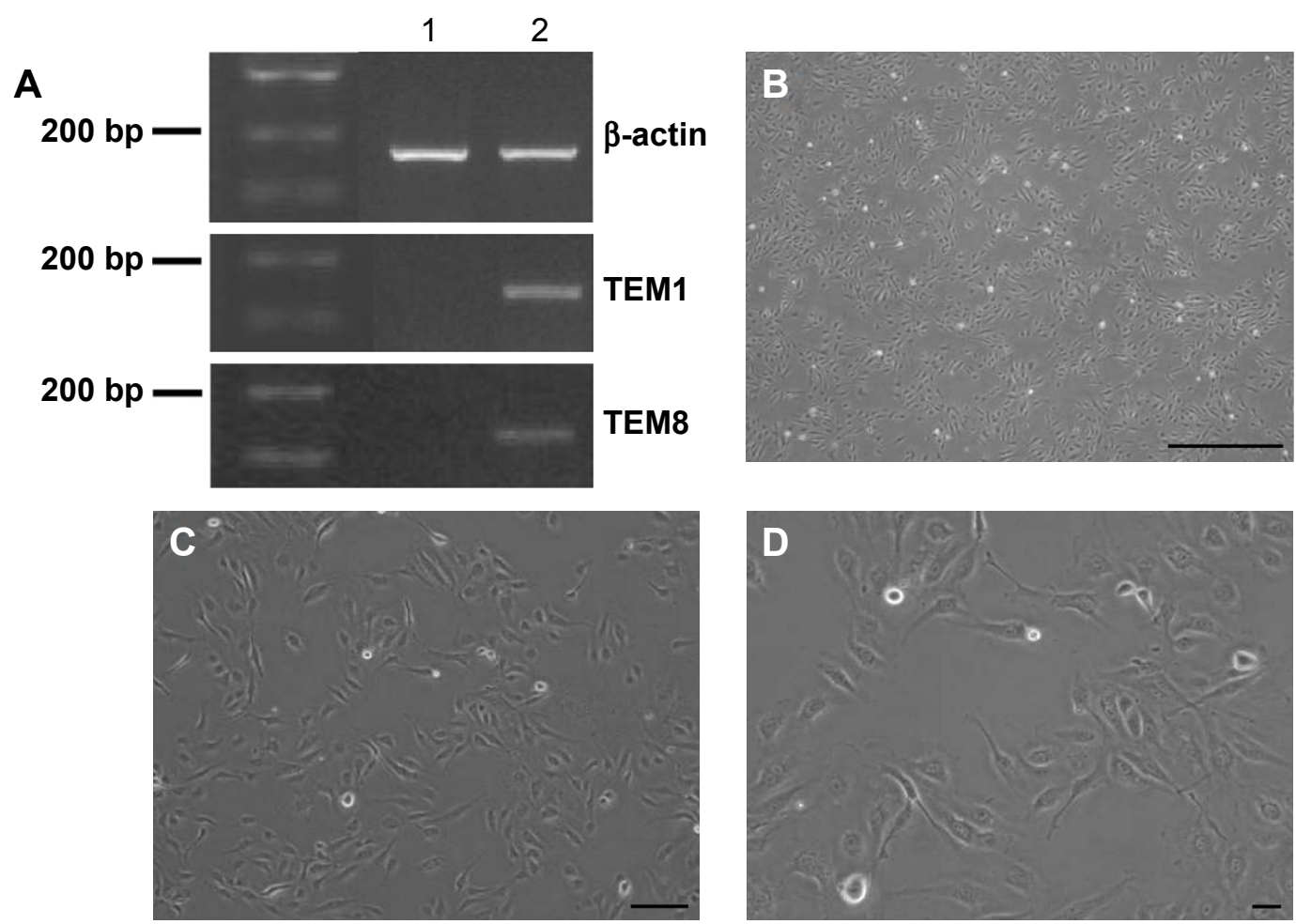

Figure I Td-EC detection and cell morphology.

Notes: (A) TEMI and TEM 8 gene expression in HUVEC and Td-EC was detected by PCR; I) HUVEC, 2) Td-EC. The results are shown by agarose gel electrophoresis; $\beta$-actin served as internal control. Representative of Td-EC cell morphology at different magnifications, (B) Magnification 40×. (C) Magnification I00×. (D) Magnification 200×. Scale bars $=100 \mu \mathrm{m}$.

Abbreviations: HUVEC, human umbilical vein endothelial cells; PCR, polymerase chain reaction; Td-EC, tumor-derived endothelial cells; TEM, tumor endothelial marker.

\section{Discussion}

$\mathrm{HCC}$ is one of the most predominant malignancies with high fatality rate, leading to over 600,000 deaths annually. ${ }^{13}$ The treatment modalities for HCC include surgical resection, radiofrequency ablation, and liver transplantation.

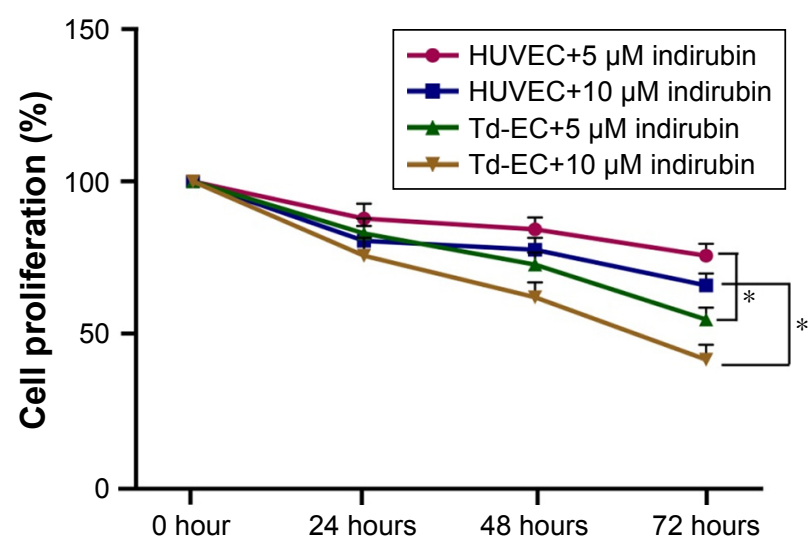

Figure 2 Effects of indirubin on cell proliferation.

Notes: HUVEC and Td-EC were treated with indirubin at different concentrations $(5,10 \mu \mathrm{M})$ for different time periods $(24,48$ and 72 hours). Dimethylsulfoxide was used as a negative control. All the quantitative values are presented as means $\pm \mathrm{SEM}$. $* P<0.05$.

Abbreviations: HUVEC, human umbilical vein endothelial cells; SEM, standard error of the mean; Td-EC, tumor-derived endothelial cells.
Unfortunately, HCC frequently recurs. ${ }^{14}$ Therefore, a growing number of researchers are seeking new treatments for HCC and focus on TCM. TCM, a complete system of healing developed in ancient China, has been receiving more and more attention in China, and throughout the world in recent decades. ${ }^{15}$ TCM is considered a medical system for the prevention and treatment of diseases that focuses on the patient rather than the disease, when compared to the Western medicine ${ }^{16}$ In this study, we focus on indirubin, the major active anti-tumor component of a traditional Chinese herbal medicine used for treatment of chronic myelogenous leukemia. However, there is little research about indirubin's effects on HCC.

The stroma is known to maintain the tissue homeostasis and acts as a barrier toward tumor formation. However, when a cell becomes malignant, its surrounding matrix changes in a way to support cancer development. ${ }^{17}$ Thus, understanding the tumor microenvironment (TME) has become as important as the modulation of cancer cell progression itself. ${ }^{18}$ The architecture of a typical TME is composed of fibroblasts, myofibroblasts, endothelial cells, pericytes, adipose cells, immune and inflammatory cells, and the extracellular matrix elements. ${ }^{19}$ In this research, we pay close attention to Td-EC, which are 

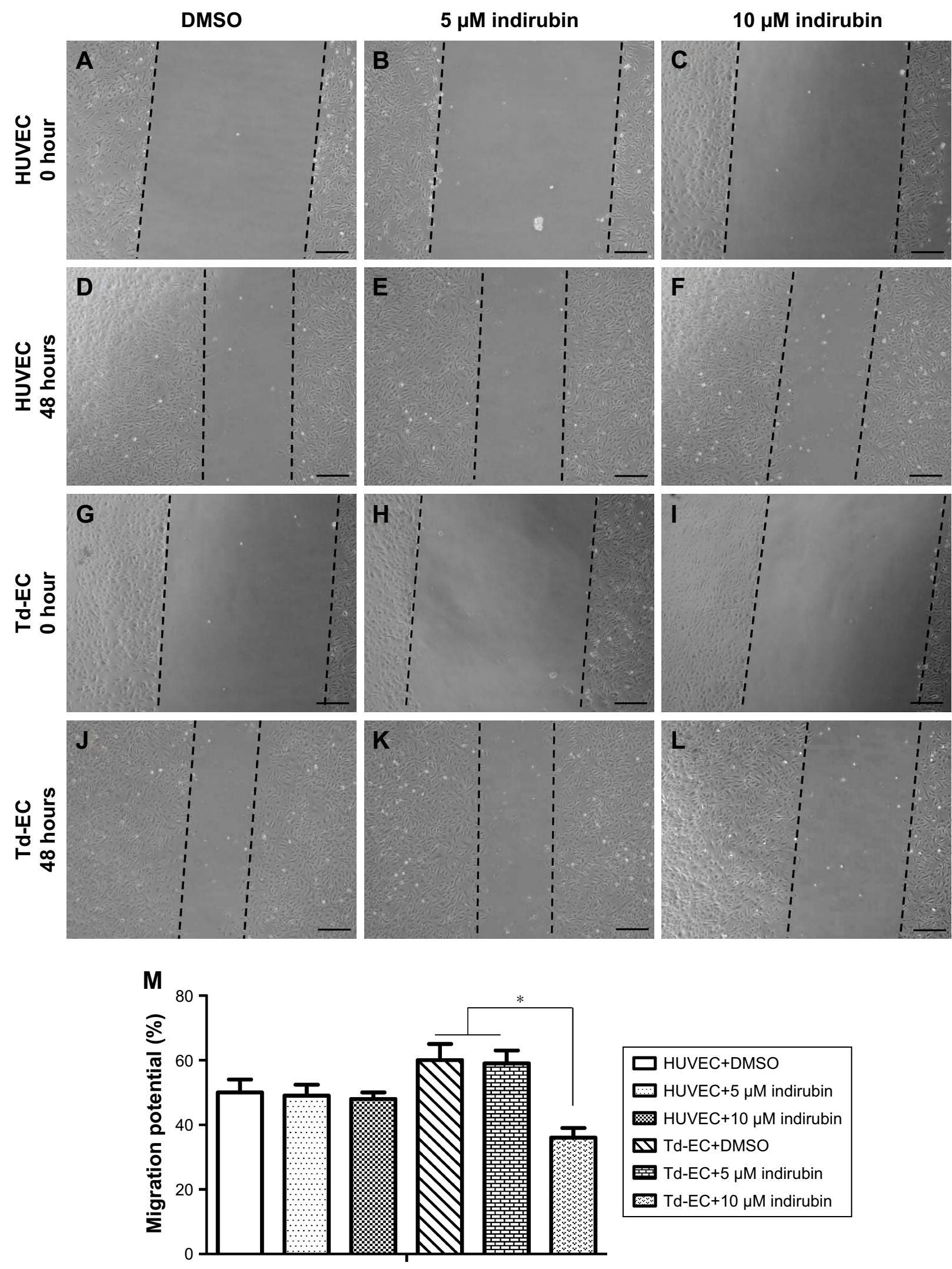

48 hours

Figure 3 Effects of indirubin on Td-EC migration ability in vitro.

Notes: Wounding healing assay detected $5 \mu \mathrm{M}$ indirubin's effects on HUVEC (A-F) and Td-EC (G-L) migration at 48 hours. DMSO was used as a negative control. Magnification 100x. Scale bars $=100 \mu \mathrm{m}$. Quantification of migration potential (\%) is shown in (M). $* P<0.05$.

Abbreviations: DMSO, dimethylsulfoxide; HUVEC, human umbilical vein endothelial cells; Td-EC, tumor-derived endothelial cells. 

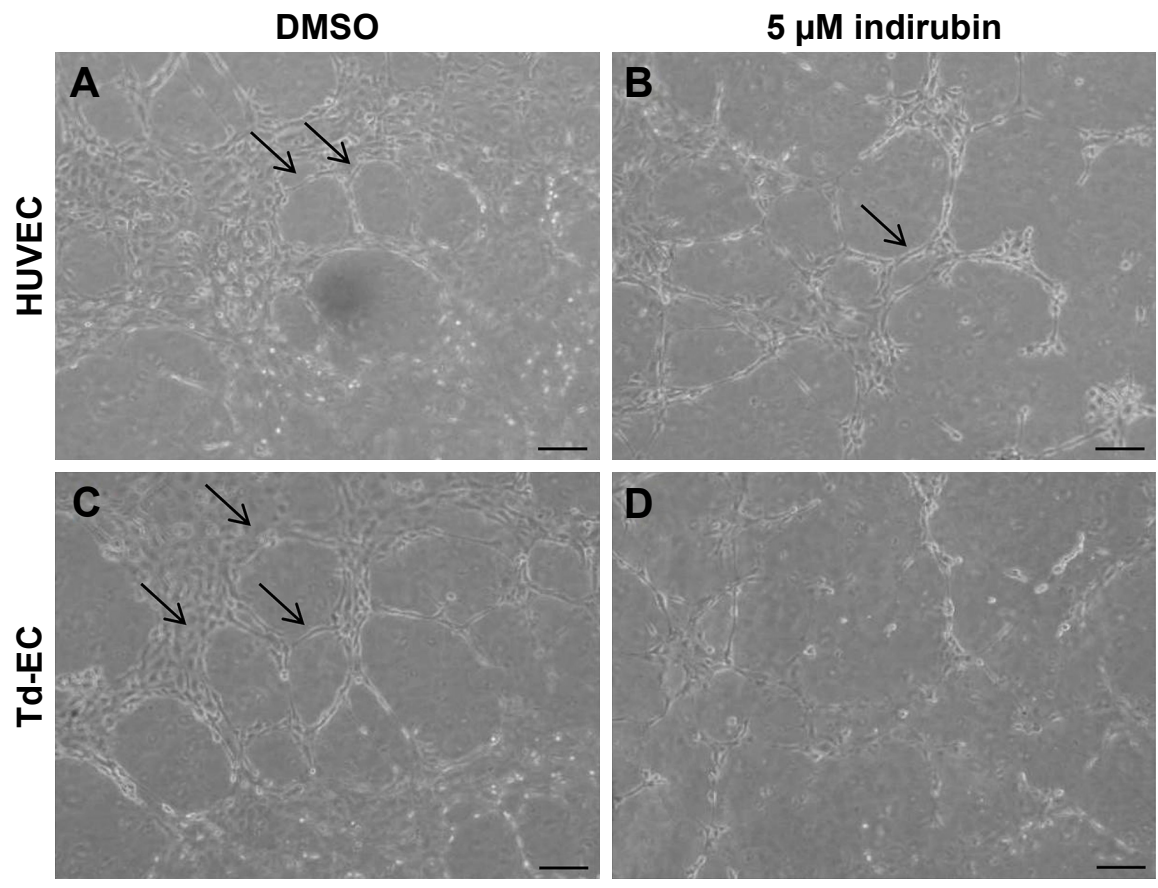

$\mathbf{E}$

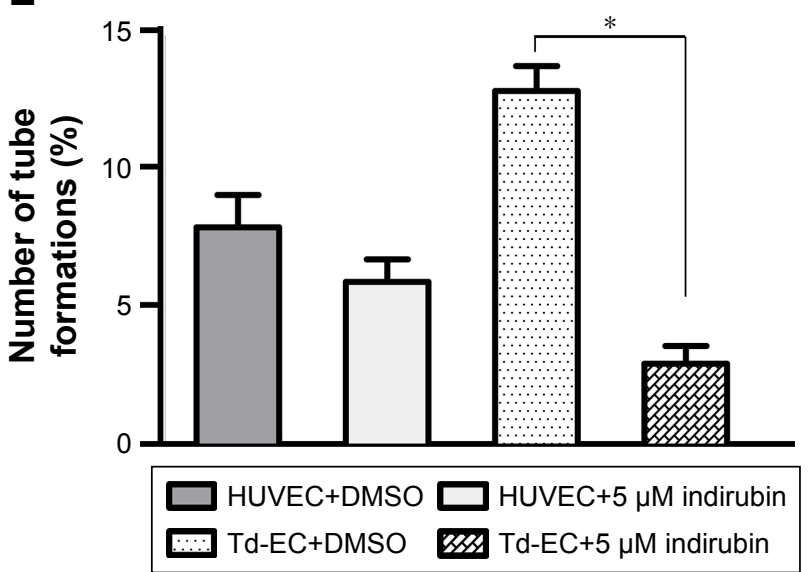

Figure 4 Effects of indirubin on Td-EC tube formation in vitro.

Notes: HUVEC (A, B) and Td-EC (C, D) were treated with $5 \mu \mathrm{M}$ indirubin. DMSO was used as a negative control. The tube-like structures are indicated by black arrows. Magnification 100×. Scale bars $=100 \mu \mathrm{m}$. Quantification of tube formation number (\%) is shown in $(E) . * P<0.05$.

Abbreviations: DMSO, dimethylsulfoxide; HUVEC, human umbilical vein endothelial cells; Td-EC, tumor-derived endothelial cells.

surrounded by other cells such as pericytes that make them more stable and control the vessel diameter and elasticity as well. ${ }^{20}$ Metastasis and angiogenesis are among the hallmarks of malignant behavior of cancer cells. Metastasis is responsible for the rapid progress and death by HCC. Angiogenesis and tumor progression are very closely linked to each other. Tumor cells are dependent on angiogenesis because their growth and expansion require oxygen and nutrients. ${ }^{21}$

TEM1 expressed in tumor endothelium but not in normal endothelium, was recently identified as a novel tumor endothelial cell surface marker potentially involved in angiogenesis. ${ }^{22}$ TEM8 is considered as a novel extracellular tumor marker among the other cell surface TEMs. TEM8 expression pattern is tumor-specific and has not been detected in physiologic angiogenesis. ${ }^{23}$ Thus, in this study, we chose TEM1 and TEM8 for Td-EC validation.

In our study, we found that indirubin inhibited cell proliferation, migration, invasion and angiogenesis of Td-EC. All these data suggest that indirubin may be a novel antiangiogenic treatment for HCC. There are many molecular mechanisms for Td-EC angiogenesis such as tumor hypoxia, epithelial-mesenchymal transition, cancer stem-like cells, and some transcription factors. More molecular mechanisms shall be identified in future indirubin studies. 

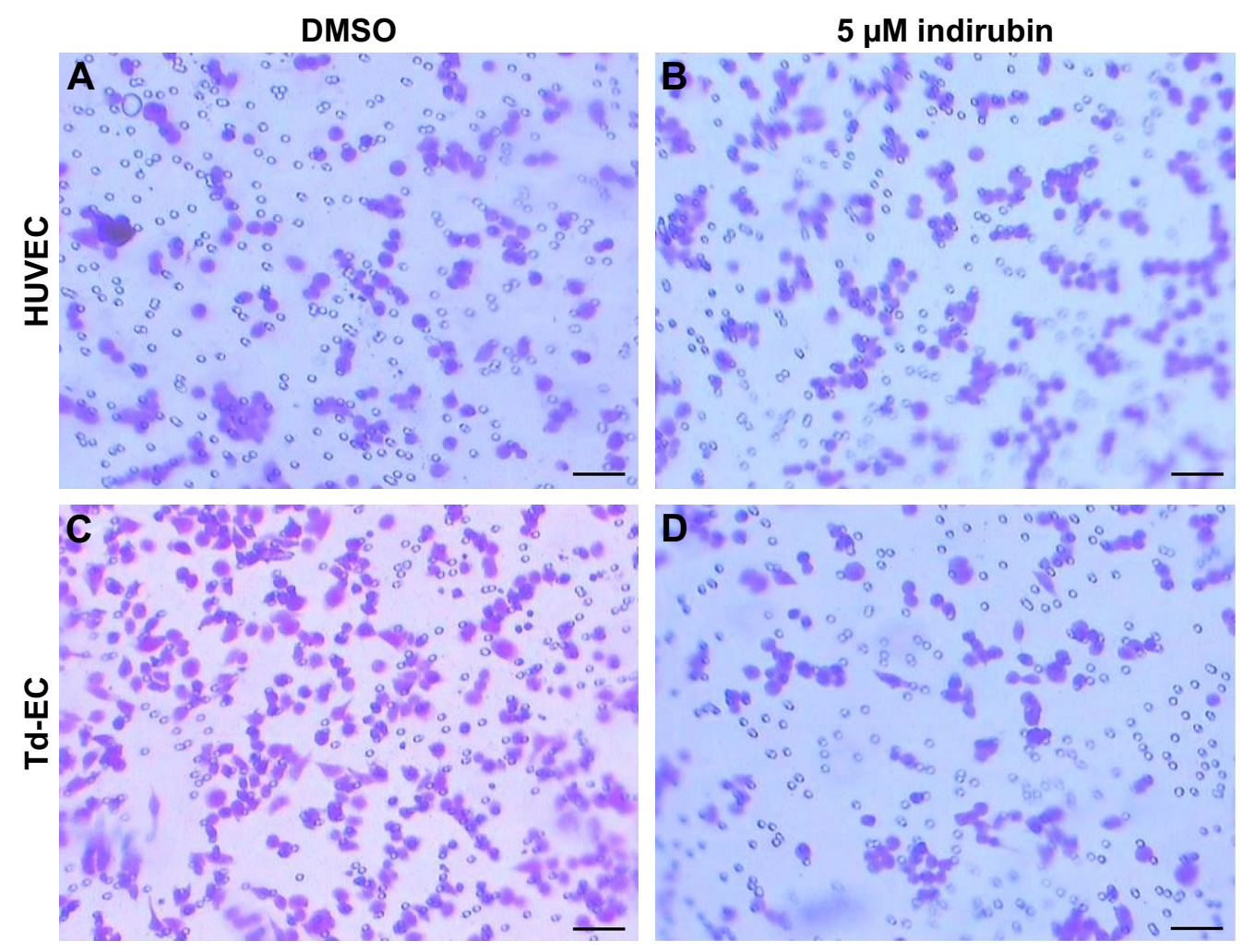

E

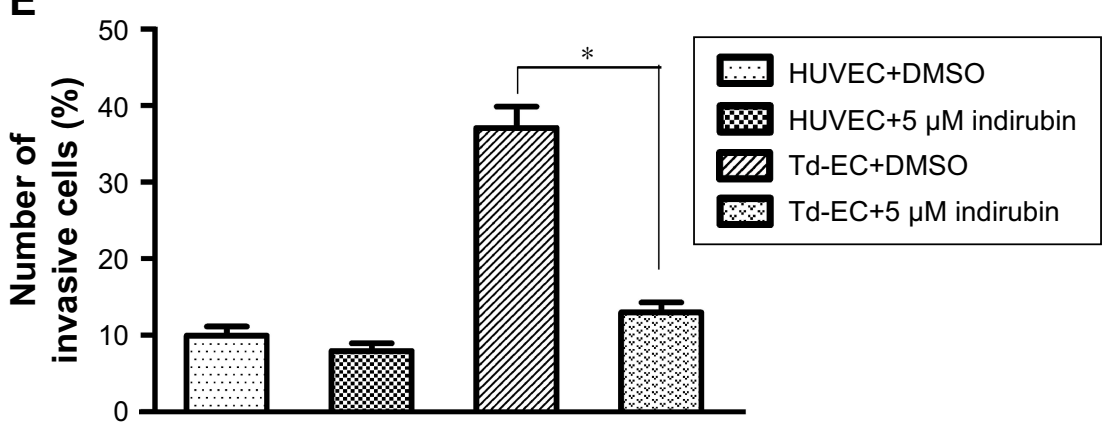

Figure 5 Effects of indirubin on cell invasion ability in vitro.

Notes: HUVEC (A, B) and Td-EC (C, D) were treated with $5 \mu$ M indirubin. DMSO was used as a negative control. Magnification I00x. Scale bars $=100 \mu m$. Quantification of invasive cells number (\%) is shown in (E). $* P<0.05$.

Abbreviations: DMSO, dimethylsulfoxide; HUVEC, human umbilical vein endothelial cells; Td-EC, tumor-derived endothelial cells.

\section{Conclusion}

Our findings indicate that indirubin can inhibit Td-EC proliferation, migration, invasion, and angiogenesis. All these suggest that indirubin can be used in the anti-angiogenic treatment of human HCC.

\section{Acknowledgment}

The present study was supported by a Scientific and Technological Development Fund (\#2013-0-yy-02) from the Affiliated Hospital of Chengdu University of TCM.

\section{Disclosure}

The authors report no conflicts of interest in this work.

\section{References}

1. Ferlay J, Soerjomataram I, Dikshit R, et al. Cancer incidence and mortality worldwide: sources, methods and major patterns in GLOBOCAN 2012. Int J Cancer. 2015;136(5):E359-E386.

2. Shojaei F. Anti-angiogenesis therapy in cancer: current challenges and future perspectives. Cancer Lett. 2012;320(2):130-137.

3. Wei Y, Yang Q, Zhang Y, et al. Plumbagin restrains hepatocellular carcinoma angiogenesis by suppressing the migration and invasion of tumor-derived vascular endothelial cells. Oncotarget. 2017;8(9): 15230-15241.

4. Rmali KA, Puntis MC, Jiang WG. Prognostic values of tumor endothelial markers in patients with colorectal cancer. World J Gastroenterol. 2005;11(9):1283-1286.

5. Tomkowicz B, Rybinski K, Foley B, et al. Interaction of endosialin/TEM1 with extracellular matrix proteins mediates cell adhesion and migration. Proc Natl Acad Sci U S A. 2007;104(46):17965-17970. 
6. Nanda A, Karim B, Peng Z, et al. Tumor endothelial marker 1 (Tem1) functions in the growth and progression of abdominal tumors. Proc Natl Acad Sci U S A. 2006;103(9):3351-3356.

7. Yang M, Chaudhary A, Seaman S, et al. The cell surface structure of tumor endothelial marker 8 (TEM8) is regulated by the actin cytoskeleton. Biochim Biophys Acta. 2011;1813(1):39-49.

8. Fu S, Tong X, Cai C, et al. The structure of tumor endothelial marker 8 (TEM8) extracellular domain and implications for its receptor function for recognizing anthrax toxin. PLoS One. 2010;5(6):e11203.

9. Tomita Y, Dorward H, Yool AJ, et al. Role of aquaporin 1 signalling in cancer development and progression. Int J Mol Sci. 2017;18(2):E299.

10. Wahid B, Ali A, Rafique S, Idrees M. New insights into the epigenetics of hepatocellular carcinoma. Biomed Res Int. 2017;2017:1609575.

11. Xiao Z, Hao Y, Liu B, Qian L. Indirubin and meisoindigo in the treatment of chronic myelogenous leukemia in China. Leuk Lymphoma. 2002;43(9):1763-1768.

12. Pietrzyk Ł. Biomarkers discovery for colorectal cancer: a review on tumor endothelial markers as perspective candidates. Dis Markers. 2016;2016:4912405.

13. Chen W, Zheng R, Baade PD, et al. Cancer statistics in China, 2015. CA Cancer J Clin. 2016;66(2):115-132.

14. Grandhi MS, Kim AK, Ronnekleiv-Kelly SM, Kamel IR, Ghasebeh MA, Pawlik TM. Hepatocellular carcinoma: from diagnosis to treatment. Surg Oncol. 2016;25(2):74-85.

15. Yu F, Takahashi T, Moriya J, et al. Traditional Chinese medicine and Kampo: a review from the distant past for the future. J Int Med Res. 2006;34(3):231-239.
16. Sánchez-Vidaña DI, Rajwani R, Wong MS. The Use of Omic Technologies Applied to Traditional Chinese Medicine Research. Evid Based Complement Alternat Med. 2017;2017:6359730.

17. Shimoda M, Mellody KT, Orimo A. Carcinoma-associated fibroblasts are a rate-limiting determinant for tumour progression. Semin Cell Dev Biol. 2010;21(1):19-25.

18. Pavlova NN, Thompson CB. The emerging hallmarks of cancer metabolism. Cell Metab. 2016;23(1):27-47.

19. Chen F, Zhuang X, Lin L, et al. New horizons in tumor microenvironment biology: challenges and opportunities. BMC Med. 2015;13:45.

20. Dudley AC. Tumor endothelial cells. Cold Spring Harb Perspect Med. 2012;2(3):a006536.

21. Chen HF, Wu KJ. Endothelial transdifferentiation of tumor cells triggered by the Twist1-Jagged1-KLF4 Axis: relationship between cancer stemness and angiogenesis. Stem Cells Int. 2016;2016:6439864.

22. Opavsky R, Haviernik P, Jurkovicova D, et al. Molecular characterization of the mouse Tem1/endosialin gene regulated by cell density in vitro and expressed in normal tissues in vivo. J Biol Chem. 2001;276(42): 38795-38807.

23. Rmali KA, Puntis MC, Jiang WG. TEM-8 and tubule formation in endothelial cells, its potential role of its vW/TM domains. Biochem Biophys Res Commun. 2005;334(1):231-238.
OncoTargets and Therapy

\section{Publish your work in this journal}

OncoTargets and Therapy is an international, peer-reviewed, open access journal focusing on the pathological basis of all cancers, potential targets for therapy and treatment protocols employed to improve the management of cancer patients. The journal also focuses on the impact of management programs and new therapeutic agents and protocols on

\section{Dovepress}

patient perspectives such as quality of life, adherence and satisfaction. The manuscript management system is completely online and includes a very quick and fair peer-review system, which is all easy to use. Visit http://www.dovepress.com/testimonials.php to read real quotes from published authors. 